This item was submitted to Loughborough's Research Repository by the author.

Items in Figshare are protected by copyright, with all rights reserved, unless otherwise indicated.

\title{
Ripple structures on ion bombarded surfaces arising from the sputter yield dependence on incidence angle
}

PLEASE CITE THE PUBLISHED VERSION

http://dx.doi.org/10.1016/j.nimb.2014.11.104

PUBLISHER

(C) Elsevier

VERSION

AM (Accepted Manuscript)

\section{PUBLISHER STATEMENT}

This work is made available according to the conditions of the Creative Commons Attribution-NonCommercialNoDerivatives 4.0 International (CC BY-NC-ND 4.0) licence. Full details of this licence are available at: https://creativecommons.org/licenses/by-nc-nd/4.0/

\section{LICENCE}

CC BY-NC-ND 4.0

\section{REPOSITORY RECORD}

Smith, Roger. 2019. "Ripple Structures on Ion Bombarded Surfaces Arising from the Sputter Yield Dependence on Incidence Angle". figshare. https://hdl.handle.net/2134/16930. 


\title{
Ripple structures on ion bombarded surfaces arising from the sputter yield dependence on incidence angle
}

\author{
Roger Smith \\ Department of Mathematical Sciences, Loughborough University, Leicestershire, LE11 \\ 3TU, UK
}

\begin{abstract}
It is shown that ripple structures on oblique incidence ion bombarded surfaces can be stable features under ion erosion without the necessity to invoke mass redistribution, surface diffusion or micro-roughening due to surface curvature dependent energy deposition. Instead the patterns are predicted to be a natural consequence of non-linear effects due to the dependence of the sputtering yield on the angle of incidence.
\end{abstract}

Keywords: ripples, ion bombardment, oblique incidence, non-linear erosion model;

\section{Introduction}

Ion-induced ripple pattern formation on semiconductor surfaces under oblique incidence low energy impact is an important phenomenon which has been used to fabricate reproducible patterns in nanotechnology for a number of years [1] but even now the basic formation mechanism is still not fully understood. One of the earliest models of ripple pattern formation was due to Bradley and Harper [2] and since then many other models have been proposed $[3,4,5,6,7,8,9,10]$. Some of these models assume that ripple patterns could form as a result of the competing effects of surface diffusion (smoothing) and micro-roughening following the Sigmund model [11]. In the Sigmund model roughening occurs because energy is deposited closer to a surface in regions where there are troughs on a surface compared to regions

$U R L:$ r.smith@lboro.ac.uk (Roger Smith) 
of positive curvature. Sigmund was able to argue that this meant that cones should preferentially form on ion bombarded surfaces as a result.

However this curvature dependent energy deposition should be a second order effect compared to the first order effect of the sputtering yield dependence on the incidence angle. If the primary effect of incidence angle dependence only is taken into account, then work in the 1970s and 1980s $[12,13,14,15]$ showed that although cones and edges can form on surfaces subjected to normal incidence ion beams, the surfaces would ultimately flatten due to the sides of the cones eroding at a faster rate than the surrounding flat surface. This is in direct contradiction to the Sigmund's micro-roughening argument.

Carter and Vishnyakov [3] first proposed that instead of the micro-roughening proposal, mass redistribution could be the mechanism by which surface features may be formed and this argument has also been used by Madi et al [17] and by Numazawa and Smith [18] to show that ripple features can arise purely as a result of mass redistribution alone without even the necessity to use erosion in the argument. In the case of [18] a travelling wave solution was found for a specific incidence angle for the non-linear equations of motion, in the form of a cycloid which matched experimental observations very well.

In this paper an even simpler two-dimensional model is introduced which shows that stable patterns can arise purely as a result of the non-linear dependence of sputtering yield on the ion incidence angle and without the need to invoke any other physical property. This effect was overlooked in the 1970's and 1980's when the theory of surface erosion was first developed because the models were generally formulated by assuming that the ion beam was incident in the direction of the average surface normal. Here we reformulate the equations for non-normal incidence and apply the model to illustrate how stable ripple-like patterns can arise.

\section{The model}

The co-ordinate system is defined in Fig. 1a. The Cartesian $x-y$ system defines directions respectively parallel and perpendicular to the average surface tangent. The gradient of the surface is given by $\tan \theta$, where $\theta$ is also the angle between the surface normal and the $y$-direction; $\phi$ is the angle between the ion beam and the surface normal while the angle $\psi$ defines the angle between the ion beam and the $y$-direction. In Fig. 1b the sputter yield dependence on incidence angle $\phi$ is plotted with a fit to $1 \mathrm{keV}$ ion bombardment 
of silicon [19]. Although this dependence is typical for many amorphous and semiconductor materials, for metals there are many more maxima and minima due to channelling [20] and also metals retain their crystallinity under ion bombardment [21] whereas silicon becomes amorphous.
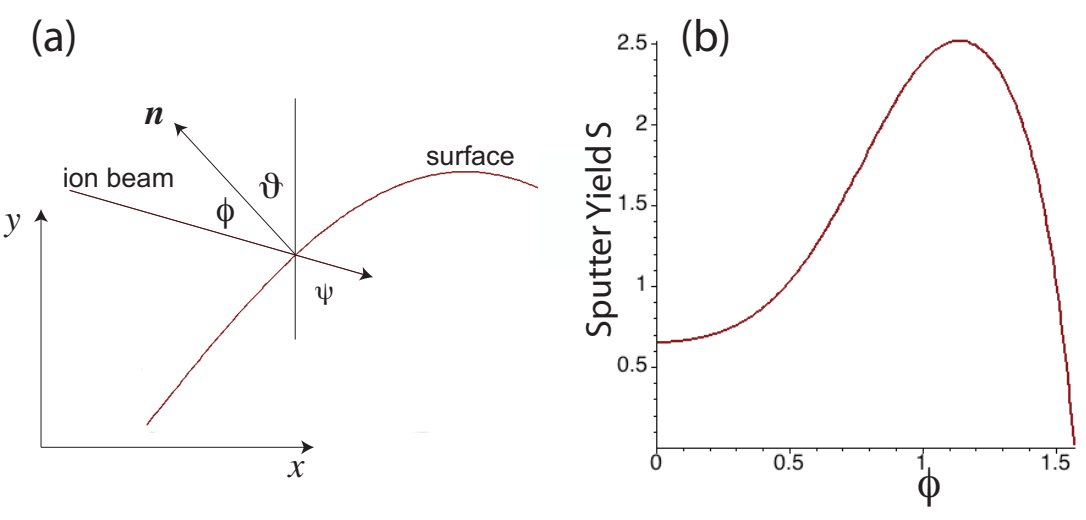

Fig. 1: (a) A description of the co-ordinate system and angles used in the calculation. The $y$ direction defines the normal to the originally flat surface. (b) The dependence of sputtering yield $S$ (atoms ejected per incident ion) on incidence angle for $1 \mathrm{keV} \mathrm{Ar}$ bombardment of Si taken from [19]. The value of $\phi$ corresponding to the maximum sputtering yield is defined as $\phi_{\max }$ which for many materials is an angle between $50^{\circ}$ and $70^{\circ}$.

If it is assumed that the rate of erosion in the ion beam direction is given by $S(\phi)$ where the flux and atom density are assumed incorporated into a non-dimensionalised time, then it easy to show, following the methodology given in [22] and [13] that the erosion can be described by two first order quasi-linear partial differential equations for $\theta$ given by

$$
\frac{\partial \theta}{\partial t}+\left(\sin \psi S(\psi-\theta)-\cos \theta \cos (\psi-\theta) S^{\prime}(\psi-\theta)\right) \frac{\partial \theta}{\partial x}=0
$$

and

$$
\frac{\partial \theta}{\partial t}-\left(\sin \theta \cos (\psi-\theta) S^{\prime}(\psi-\theta)+\cos \psi\right) S(\psi-\theta) \frac{\partial \theta}{\partial y}=0
$$

where the primes denote differentiation. In the first equation the partial differential with respect to $t$ means at constant $x$, whereas in the second equation $y$ is kept constant. These equations have been intensively investigated for the case $\psi=0[12,13,14,15]$ but in the case of ripple formation $\psi \neq 0$. The equations are of a form first investigated by Lagrange [22] and in the 1950's a similar set was used by Lighthill and Whitham [23] to model the 
flow of traffic. The method of solution involves integration along the characteristic curves [22] but since the right hand sides of the equations are zero, the characteristic curves define straight lines of constant surface orientation $(\theta=$ const $)$. The characteristic curves are given in the usual way [22] by the equations

$$
\frac{d x}{d t}=\sin \psi S(\psi-\theta)-\cos \theta \cos (\psi-\theta) S^{\prime}(\psi-\theta)
$$

and

$$
\frac{d y}{d t}=-\sin \theta \cos (\psi-\theta) S^{\prime}(\psi-\theta)-\cos \psi S(\psi-\theta)
$$

These lines have gradient given by

$$
\begin{aligned}
\frac{d y}{d x}= & \left(-\sin \theta \cos (\psi-\theta) S^{\prime}(\psi-\theta)-\cos \psi S(\psi-\theta)\right) \\
& /\left(\sin \psi S(\psi-\theta)-\cos \theta \cos (\psi-\theta) S^{\prime}(\psi-\theta)\right) .
\end{aligned}
$$

Thus the surface evolution can be plotted graphically in the same way as the Huyghen's wave front construction in geometrical optics, except that now the wavefront does not propagate isotropically. Instead parts of the surface expand into facets, whereas other parts of the surface contract into edges or shocks (discontinuities in $\theta$ ). The shocks are equivalent to caustic curves in geometrical optics [24]. To examine which parts of the surface expand and which parts contract, consider a small element of the initial surface $\delta s_{0}$. After time $t$, using equations 3 and 4 it is possible to evaluate how $\delta s_{0}$ evolves . After some algebra the corresponding element, $\delta s$, on the evolved surface can be calculated as

$$
\begin{aligned}
\delta s^{2}= & \delta s_{0}^{2}\left(1+2 t \kappa\left[\cos (\psi-\theta) S^{\prime \prime}(\psi-\theta)\right.\right. \\
& \left.\left.-2 \sin (\psi-\theta) S^{\prime}(\psi-\theta)\right]\right) \\
& \times\left[1+t \kappa\left[\cos (\psi-\theta) S^{\prime \prime}(\psi-\theta)-\right.\right. \\
& \left.2 \sin (\psi-\theta) S^{\prime}(\psi-\theta)\right] .
\end{aligned}
$$

Here $\kappa$ is the curvature of the surface and is a function of $\theta$. Thus expansion waves occur when the function $g(\theta, \psi)$ is positive where

$$
g(\theta, \psi)=\kappa\left[\cos (\psi-\theta) S^{\prime \prime}(\psi-\theta)-2 \sin (\psi-\theta) S^{\prime}(\psi-\theta)\right] .
$$

Equation (7) was also derived in [12] when $\psi=0$ and an equivalent expression by Budil and Hobler [16]. 


\section{Results and Discussion}

One can now see why this formulation is in contradiction to the microroughening argument of Sigmund for normally incident ion beams $(\psi=0)$ by using a specific example. Consider a surface initially described by a simple sinusoidal curve subject to erosion by a normally incident beam shown in Fig. 2 for different times. One can see how the surface maxima evolve into discontinuities due to the collapse of surface elements $(\kappa<0)$, whereas the bottom of the valleys expand $(\kappa>0)$. The sides of the 'cones' evolve into facets corresponding to the maxima $\left(g_{\theta}=0\right)$ in equation 7 before the surface eventually flattens due to these facets eroding more quickly than the flat concave areas of the surface. This rather remarkably depends on the third derivative (the maximum occurs when $g_{\theta}=0$ ) of the sputtering yield with respect to the incidence angle and the surface curvature defines whether or not locally flat regions either sharpen (tops of ridges) or expand (bottoms of valleys). Facets therefore do not always form corresponding to planes where the sputtering yield is a maximum as was predicted over 40 years ago by Stewart and Thompson [25]. Conical structures do form and persist on ion-bombarded surfaces due to crystallinity effects such as grain boundaries or due to impurities shielding parts of a surface that erode at a faster rate. Indeed similar structures can also be seen in geology such as at Tent Rocks in New Mexico. However any initially flat surface under bombardment by a normally incident beam with the sputtering yield-angle of incidence dependence as given in Fig. 1b will remain flat since any perturbations in the surface height will flatten as the result in Fig.2 demonstrates.

Now consider a similar situation when the beam is incident with $\psi=75^{\circ}$ on a surface $y=\sin 0.262 x$. The value of 0.262 was chosen so that maximum negative gradient on the trailing slopes of the sine wave were approximately aligned with the incoming beam. The result is shown in Fig. 3. In this case the initial undulations grow slightly with the front side of the ripples developing an orientation equal to the maximum gradient of the initial sinusoidal structure and the trailing edges parallel to the characteristic curve corresponding to that orientation. The steady state structure is a rippled surface with the same wavelength as the initial undulations but with an enhanced height. Note that this situation only arises because all the characteristics have negative slope and all the surface except that which has the maximum gradient collapses into the edge at the top of the structure. At lower angles of incidence the smoothing process occurs similar to that shown in Fig.2. 
(a)

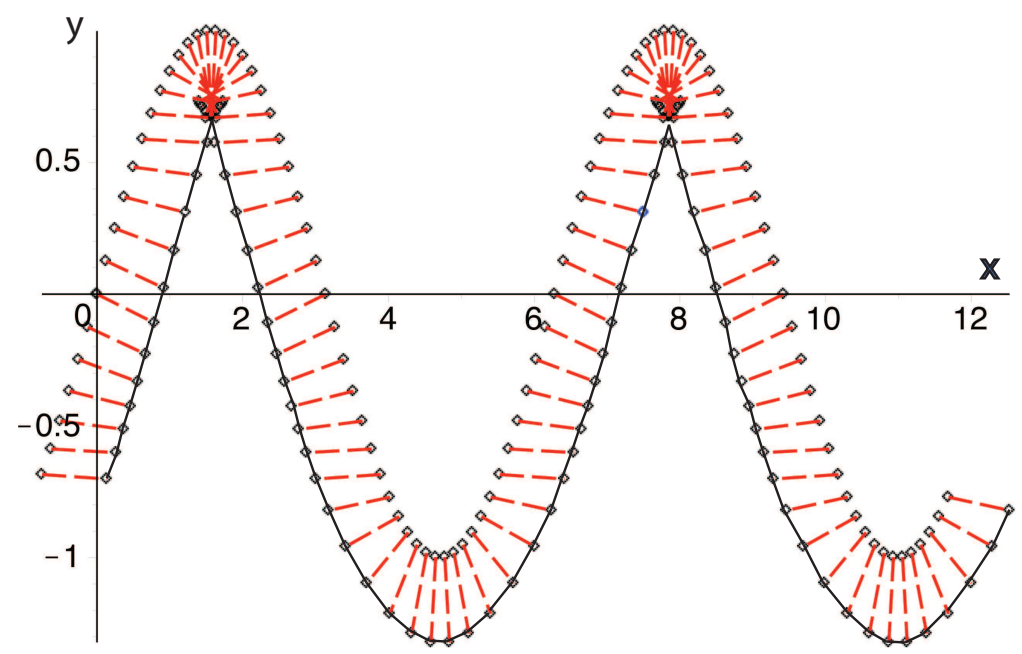

(b)

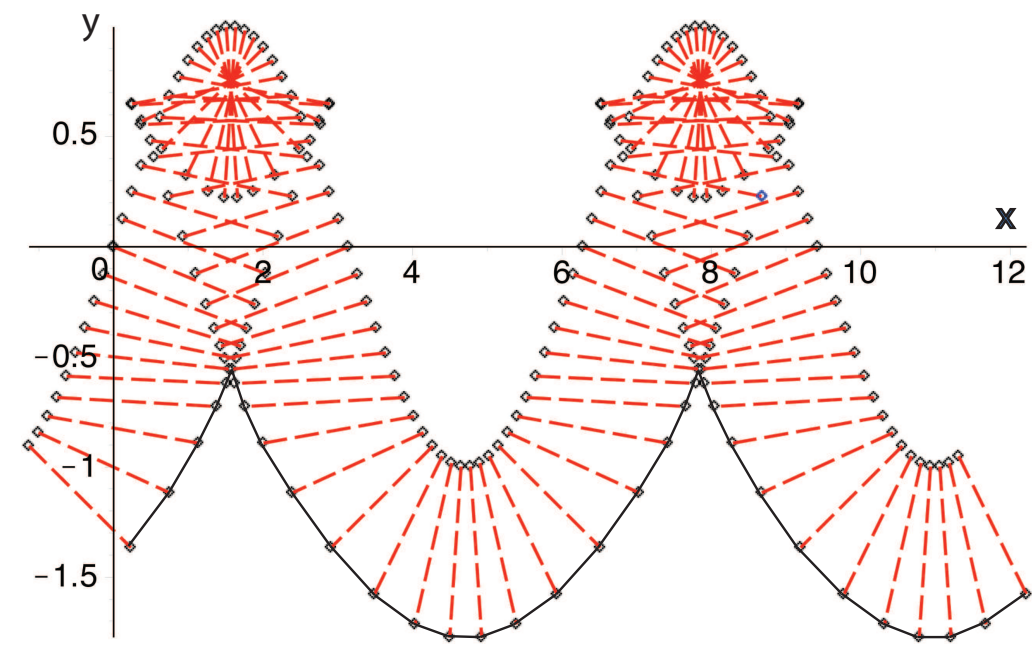

Fig. 2: The erosion of the sinusoidal curve $y=\sin x$ when the beam is incident along the $y$-direction with the angular yield dependence given by the curve shown in Fig. $1 \mathrm{~b}$ after 2 different times (a) $t=0.5$ and (b), $t=1.2$. The dashed curves are the characteristic lines. The solid curve is the envelope of the evolved surface. 
(a)

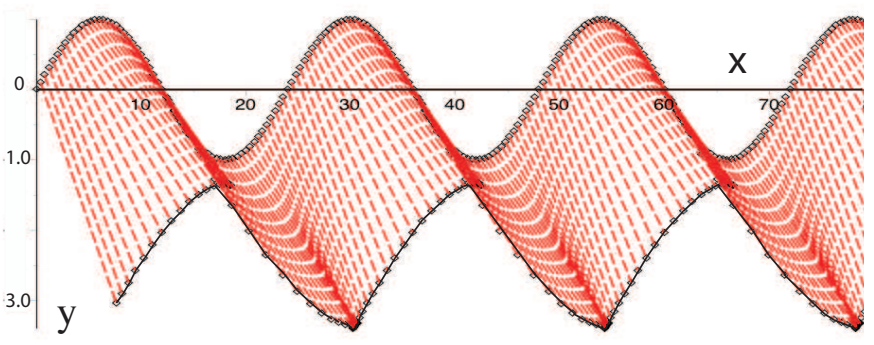

(b)

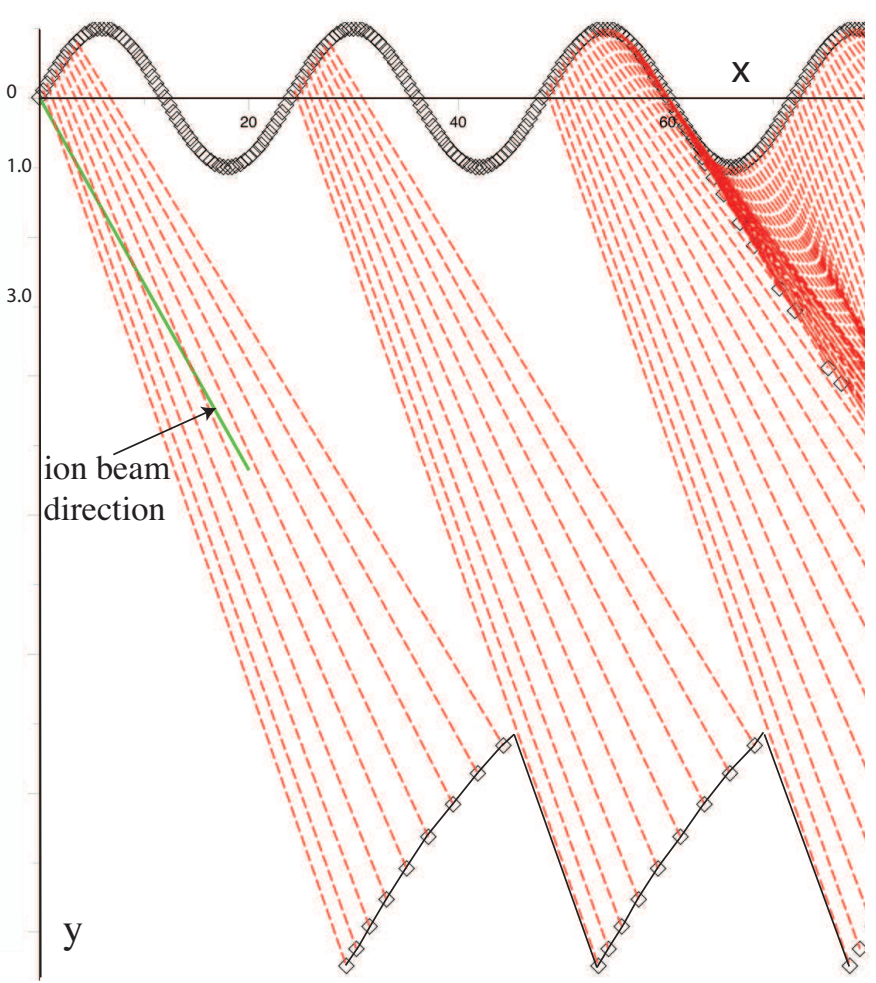

Fig. 3: The erosion of the sinusoidal curve $y=\sin 0.262 x$ incident at $\psi=75^{\circ}$ with the angular yield dependence given by the curve shown in Fig. 1b after 2 different times (a) $t=4.0$ and (b), $t=16$. The dashed curves are the characteristic lines. The solid curve is the envelope of the evolved surface. 


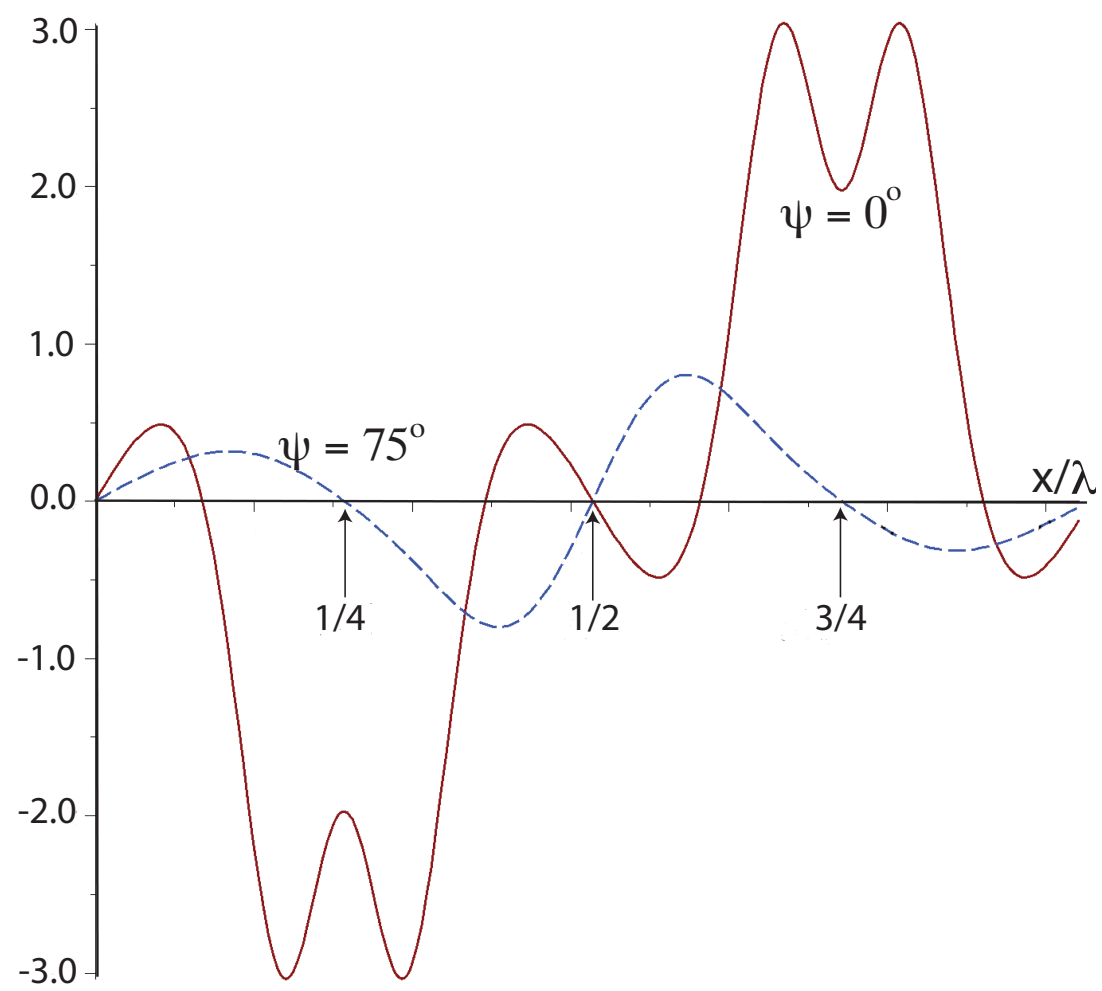

Fig. 4: The function $g(\theta, \psi)$ plotted as a function of wavelength for the two initial sinusoidal curves shown in Figs. $2(\psi=0)$ and $3\left(\psi=75^{\circ}\right)$. The unbroken line refers to Fig. 2 and the dashed line refers to Fig. 3. 
Fig. 4 gives the function $g$ plotted as a function of wavelength for the two initial sinusoidal curves shown in Figs. 2 and 3. We can see that in the case of the normally incident beam there is a region in the first half wavelength including the maximum at $x / \lambda=1 / 4$ where the characteristics converge, $g<0$, forming the fast eroding apex, whereas in the case of oblique incidence the characteristics diverge, $g>0$, in the first quarter wavelength promoting facets as seen in Fig. 3. The characteristics converge in the second quarter wavelength for oblique incidence which collapses into an edge.

Fig. 5 shows the case of a stably eroding structure where the original surface shape is preserved. In this case the beam is exactly aligned along the characteristics from plane (1) which also defines the slope of the trailing edge, labelled (2) in Fig. 5a. If the gradient of this edge is steeper so that shadowing occurs, then a facet develops at the convex apex which expands and the trailing edge will decay until it becomes parallel to the beam direction. This situation gives the maximum height that a periodic system of intersecting planes of a set wavelength $\lambda$ can attain and remain stable under further erosion. Intersecting planes of the form shown in Fig. 5 can only occur if the gradient of the characteristics from the plane with positive gradient (labelled (1)) is negative and aligned with the direction of the incoming beam. This is only possible for a small range of $\theta$ and for $\psi>\phi_{\max }$. The range of allowable values is plotted in Fig. 5b. The maximum angle for plane (1) occurs when the beam is at grazing incidence to the initial surface and $\theta=24.81^{\circ}$ for the $S(\phi)$ dependence shown in Fig. 1. The variation of plane gradient as a function of incidence angle is plotted along with the corresponding height to wavelength ratio. The example plotted in Fig. 3 for $\psi=75^{\circ}$ develops into such structures with a leading plane angle of $\theta \approx 10^{\circ}$ and a height to wavelength ratio of about 0.18 . Initially this value was 0.08 and the structures grow in height until they reach the steady state value. The stepped structures predicted in Fig. 5 are thus equivalent in some sense to the stable travelling wave solutions described in [18] but without the need to invoke mass redistribution as a mechanism for their formation.

The theory cannot explain how initial undulations in the surface height occur on a flat uniform surface since it is based on the idea that surface orientations are preserved under ion erosion but what it shows is that when certain preferred angles of orientation are formed they remain stable under erosion and in some circumstances initial undulations can be magnified. These stable structures are in some sense similar to the travelling wave solutions previously found [18] but without the need to invoke mass redistribution 

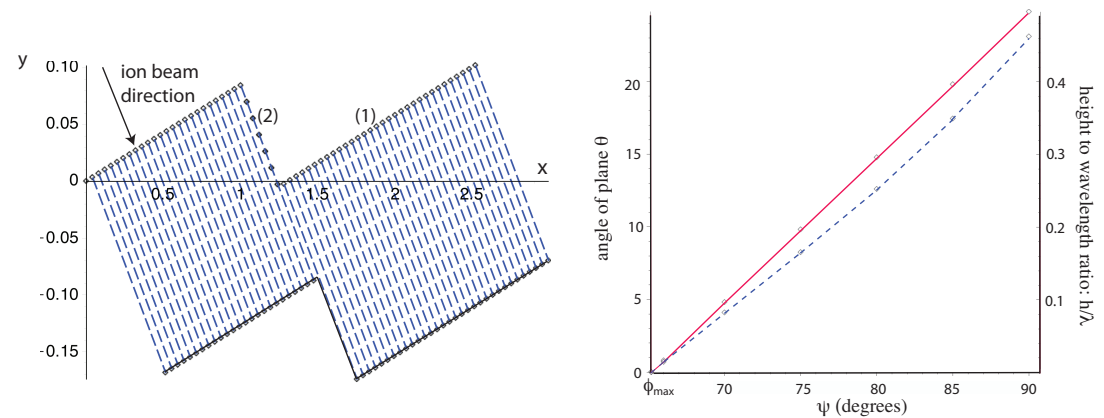

Fig. 5: (a) The erosion of intersecting planes when the beam is incident along the direction of the characteristics, for $\psi=70^{\circ}$ and $\theta=4.8^{\circ}$, when $t=0.2$. The dashed curves are the characteristic lines. (b) The allowable angles of plane 1 as a function of the beam direction $\psi$ for the situation when the beam is aligned parallel to plane 2 (solid line) for the sputter yield curve shown in Fig. 1b. The broken line gives the height to wavelength ratio of these structures as a function of incidence angle.

as a mechanism. We conclude that non-linear effects are highly significant to explain the stability of ripple patterns on ion bombarded surfaces. However one might expect that either mass redistribution or atomistic effects such as those proposed in [26] should be the important processes by which the specific surface orientations are formed and which the non-linear model described here predicts would be stabilised by bombardment.

\section{Acknowledgment}

The author thanks Karl-Heinz Heinig, Wolfhard Möller, Stefan Facsko, Satoshi Numazawa and Mukesh Ranjan for useful discussions. RS is also grateful to the Helmholtz Zentrum Dresden Rossendorf for the funding to pursue this work and to the British Council exchanges with India scheme, UKIERI Grant: IND/CONT/E/13-14/642.

[1] S. Facsko, T. Dekorsy, C. Koerdt, C. Trappe, H. Kurz, A. Vogt and H. L. Hartnagel, Science 2851551 (1999).

[2] R. M. Bradley and J. M. E. Harper, J. Vac. Sci. Technol. A 62390 (1988).

[3] G. Carter and V. Vishnyakov, Phys. Rev. B 5417647 (1996). 
[4] R. Cuerno and A. L. Barabási, Phys. Rev. Lett. 744746 (1995).

[5] M. Castro, R. Cuerno, L. Vazquez and R. Gago, Phys. Rev. Lett. 94 016102 (2005).

[6] J. Muñoz-García, R. Gago, L. Vázquez, J. A. Sánchez-García, and R. Cuerno, Phys. Rev. Lett. 104026101 (2010).

[7] M. Castro and R. Cuerno, Appl. Surf. Sci. 2584171 (2012).

[8] J. Erlebacher, M. J. Aziz, E. Chason, M. B. Sinclair and J. A. Floro, Phys. Rev. Lett. 82(11) 2330 (1999).

[9] E. Chason, J. Erlebacher, M. J. Aziz, J. A. Floro and M. B. Sinclair, Nucl. Instr. and Meth. B 17855 (2001).

[10] W. Möller, Nucl. Instr. and Meth. B 32223 (2014).

[11] P. Sigmund, J. Mater. Sci. 81545 (1973).

[12] G. Carter, J. C. Colligon and M. J. Nobes, Rad. Eff. and Defects in Solids 3165 (1977).

[13] R. Smith and J. M. Walls, Phil. Mag. A 42235 (1980).

[14] R. Smith, G. Carter and M. J. Nobes, Proc. Roy. Soc. London. A 407 405 (1986).

[15] I. V. Katardjiev, G. Carter, M. J. Nobes and R. Smith J. Vac. Sci. Tech. A 62443 (1988).

[16] M. Budil and G. Hobler Nucl. Instrum and Meth. B 2691614 (2011).

[17] C. S. Madi, E. Anzenberg, K. F. Ludwig Jr. and M. J. Aziz, Phys. Rev. Lett. 106066101 (2011).

[18] S. Numazawa and R. Smith, J. Phys. Condensed. Matt. 25095003 (2013).

[19] J. P. Ducommun, M. Cantagrel and M. Moulin, J. Mater. Sci. 1052 (1975).

[20] D. Onderdelinden, Can. J. Phys. 46739 (1967). 
[21] C. Scott and R. Smith, Proc. Roy. Soc. London. A 46920120480 (2013).

[22] L. C. Evans (1998), Partial Differential Equations, Providence: American Mathematical Society, ISBN 0-8218-0772-2

[23] M. J. Lighthill and G. B. Whitham Proc. Roy. Soc. London. A 229317 (1955).

[24] P. Ferraro The Physics Teacher 34572 (1996)

[25] A. D. G. Stewart and M. W. Thompson, J. Mater. Sci. 456 (1969).

[26] S. A. Norris, J. Samera, L. Bukonte, M. Backman, F. Djurabekova, K. Nordlund, C. S. Madi, M. P. Brenner and M. J. Aziz, Nature Comm. 2 276 (2011). 